\title{
Construction of a Unified Model for Formal Contexts and Formal Decision Contexts
}

\author{
Xia Wang \\ School of Mathematics, Physics and Information Science \\ Zhejiang Ocean University \\ Zhoushan, Zhejiang 316022, China \\ bblylm@126.com
}

\begin{abstract}
This paper mainly constructs a unified model for formal contexts, consistent formal decision contexts and inconsistent formal decision contexts based on object oriented concept lattices, which is called a consistent approximate representation space. Congruence relations are first introduced into formal contexts and then relationships between congruence relations and the corresponding object oriented concept lattices are developed. Finally, consistent approximate representation space is defined for a formal context, which is a quadruple $\left(U, A, \Re, R^{\prime}\right)$. It is verified that if we give $R^{\prime}$ different meanings, then we obtain the corresponding formal context, consistent formal decision context and inconsistent formal decision context with respect to object oriented concept lattices. Therefore, the quadruple $\left(U, A, \Re, R^{\prime}\right)$ is a unified model for formal contexts and formal decision contexts.
\end{abstract}

Keywords: Object oriented concept lattice, Formal context, Congruence relation, Approximate representation space

\section{Introduction}

Formal concept analysis, proposed by Wille in 1982 [1], is an effective tool for data analysis, knowledge representation and information management. At present, many efforts have been made to construction of concept lattice [2-5], pruning of concept lattice [6], acquisition of rules [4, 5], relationship with rough set [7-13], and applications [6, 14]. In [12], Yao compared the theory of rough sets and formal concept analysis in a common framework based on formal contexts. Particularly object oriented concept lattices and property oriented concept lattices were also constructed in [12]. In [15], an approach to knowledge reduction in the object oriented concept lattices and the property oriented concept lattices were presented which can keep all extents and their original hierarchy in a formal context. Wang et al., [16] provided another approach to attribute reduction in the object oriented concept lattices and the property oriented concept lattices, which only required preserving all extents of meet irreducible elements. Relatively knowledge reduction in classical concept lattices has much more research results. Ganter et al., [2] proposed reducible attribute and reducible object from the viewpoint of shortening lines or rows. Zhang et al., [17-20] presented an attribute reduction approach to find minimal attribute sets which can determine all extents and their original hierarchy in a formal context. Wang et al., [21] provided an approach to attribute reduction based on meet irreducible elements. Wu et al., [22] studied attribute reduction in formal contexts from the viewpoint of keeping granular structure of concept lattices. Liu et al. [23] showed an efficient post-processing method to prune redundant rules by virtue of the 
property of Galois connection, which inherently constrains rules with respect to objects. Mi et al., [24] formulated a Boolean approach to calculating all reducts of a formal context via the use of discernibility function. In [25], a rule acquisition oriented framework of knowledge reduction was proposed for real decision formal contexts and a corresponding reduction method was formulated by constructing a discernibility matrix and its associated Boolean function. In [26], based on fuzzy K-means clustering, Kumar and Srinivas proposed a method to reduce the size of the concept lattices by employing corresponding object-attribute matrix. In [27], approaches to attribute reduction in a formal context and in a consistent formal decision context were proposed by preserving congruence relation classes. Wang et al., [28] studied notions and approaches to attribute reduction in an inconsistent formal decision context based on congruence relation classes.

Since databases obtained from real world are usually very complicated, knowledge reduction in formal concept analysis becomes more complex. On the other hand, through analyzing the above methods of knowledge reduction, we know that there are different places existing in knowledge reduction of different contexts even if we use the same type of method. And it makes the application of formal concept analysis more difficult. In order to solve this problem, we construct a model to unify the formal contexts, consistent formal decision contexts and inconsistent formal decision contexts, which is a quadruple $\left(U, A, \Re, R^{\prime}\right)$. It is verified that if we give $R^{\prime}$ different meanings, then we obtain corresponding formal context, consistent formal decision context and inconsistent formal decision context. Therefore, the quadruple $\left(U, A, \Re, R^{\prime}\right)$ is a unified model for formal contexts and formal decision contexts. Therefore, we can consider methods of knowledge reduction or rule acquisition of consistent approximate representation space as a whole, which can reduce the complexities of different contexts.

The paper is organized as follows. Section 2 recalls preliminaries on formal concept analysis and dependence space. Section 3 constructs a model named consistent approximate representation space and then studies relationships between consistent approximate representation spaces and the corresponding formal contexts. Finally, Section 4 concludes the paper.

\section{Preliminaries}

Some basic notions and properties about formal concept analysis and dependence space are introduced in this section.

\subsection{Basic notions about Formal Concept Analysis}

Definition 1 ([2]) A formal context $(U, A, I)$ consists of two sets $U$ and $A$, and a relation $I \subseteq U \times A$. The elements of $U$ are called objects and the elements of $A$ are called attributes of the formal context.

For $X \subseteq U$ and $B \subseteq A$, Yao [12] defined two closure operators as follows:

$$
\begin{aligned}
& X^{\square}=\{a \in A \mid \forall x \in U, x R a \Rightarrow x \in X\}, \\
& X^{\diamond}=\{a \in A \mid \exists x \in U, x R a \wedge x \in X\}, \\
& B^{\square}=\{x \in U \mid \forall a \in A, x R a \Rightarrow x \in B\}, \\
& B^{\diamond}=\{x \in U \mid \exists a \in A, x R a \wedge a \in B\} .
\end{aligned}
$$

Definition 2 ([2]) Let $(U, A, I)$ be a formal context. The formal context $\left(U, B, I_{B}\right)$ is called a subcontext of $(U, A, I)$, where $I_{B}=I \cap(U \times B)$ for any $B \subseteq A$. 
Let ${ }^{\square_{B}}{ }^{\circ B}$ stand for the operator in the subcontext $\left(U, B, I_{B}\right)$ for any $B \subseteq A$. Clearly, for $X \subseteq U, X^{\square B}=X^{\square A} \cap B, X^{\diamond B}=X^{\diamond A} \cap B$ and $X^{\square A}=X^{\square}, X^{\diamond A}=X^{\diamond}$.

Definition 3 ([12]) An object oriented concept of a formal context $(U, A, I)$ is a pair $(X, B)$ with $X \subseteq U, B \subseteq A, \quad X=B^{\circ}$ and $B=X^{\square}$. We call $X$ the extent and $B$ the intent of the object oriented concept $(X, B)$.

The concepts of a formal context $(U, A, I)$ are partially ordered by $\left(X_{1}, B_{1}\right) \leq\left(X_{2}, B_{2}\right)$ if and only if (iff for short) $X_{1} \subseteq X_{2}$ ( iff $B_{2} \subseteq B_{1}$ ), where $\left(X_{1}, B_{1}\right)$ and $\left(X_{2}, B_{2}\right)$ are two object oriented concepts. The set of all object oriented concepts of $(U, A, I)$ partially ordered in this way is denoted by $L_{O}(U, A, I)$ and is called the object oriented concept lattice of the formal context $(U, A, I)$. The infimum and supremum are given by:

$$
\begin{aligned}
& \left(X_{1}, B_{1}\right) \wedge\left(X_{2}, B_{2}\right)=\left(\left(B_{1} \cap B_{2}\right)^{\diamond}, B_{1} \cap B_{2}\right) \\
& \left(X_{1}, B_{1}\right) \vee\left(X_{2}, B_{2}\right)=\left(X_{1} \cup X_{2},\left(X_{1} \cup X_{2}\right)^{\square}\right)
\end{aligned}
$$

We denote the extent set of $(U, A, I)$ by $L_{o U}(U, A, I)=\left\{X \mid(X, B) \in L_{O}(U, A, I)\right\}$. It is evident that $L_{O U}\left(U, D, I_{D}\right) \subseteq L_{O U}(U, A, I)$ for any $D \subseteq A$.

Proposition 1 ([12]) Let $(U, A, I)$ be a formal context, $X, X_{1}, X_{2}$ be object sets, and $B, B_{1}, B_{2}$ be attribute sets, then

$$
\begin{aligned}
& \text { (i) } X_{1} \subseteq X_{2} \Rightarrow X_{1}^{\square} \subseteq X_{2}{ }^{\square}, X_{1}{ }^{\circ} \subseteq X_{2}{ }^{\circ} \text {, } \\
& B_{1} \subseteq B_{2} \Rightarrow B_{1}^{\square} \subseteq B_{2}^{\square}, B_{1}^{\diamond} \subseteq B_{2}{ }^{\circ}, \\
& \text { (ii) } X^{\square \diamond} \subseteq X \subseteq X^{\triangleleft \square}, B^{\square \diamond} \subseteq B \subseteq B^{\diamond \square} \text {, } \\
& \text { (iii) } X^{\square \diamond \square}=X^{\square}, B^{\square \diamond \square}=B^{\square} \text {, } \\
& X^{\diamond \square \diamond}=X^{\diamond}, B^{\diamond \square \diamond}=B^{\diamond}, \\
& \text { (iv) }\left(X_{1} \cap X_{2}\right)^{\square}=X_{1}^{\square} \cap X_{2}^{\square} \text {, } \\
& \left(X_{1} \cup X_{2}\right)^{\circ}=X_{1}^{0} \cup X_{2}^{\circ}, \\
& \left(B_{1} \cap B_{2}\right)^{\square}=B_{1}^{\square} \cap B_{2}^{\square}, \\
& \left(B_{1} \cup B_{2}\right)^{\diamond}=B_{1}^{\diamond} \cup B_{2}{ }^{\diamond} \text {. }
\end{aligned}
$$

\subsection{Dependence Space based on a Formal Context}

In [29], Novotny defined a congruence relation on the attribute power set $P(A)$ and dependence space in information systems.

Definition 4 ([29]) Let $(U, A, F)$ be an information system. $\kappa$ is an equivalence relation on $P(A)$. Then, Kis called a congruence relation on $(P(A), \cup)$, whenever it satisfies the following condition: if $\left(B_{1}, C_{1}\right) \in \kappa,\left(B_{2}, C_{2}\right) \in \kappa$, then $\left(B_{1} \cup B_{2}, C_{1} \cup C_{2}\right) \in \kappa$.

Definition 5 ([29]) Let $A$ be a finite nonempty set, $K$ a congruence relation on $(P(A), \cup)$. Then the ordered pair $(A, \kappa)$ is said to be a dependence space. 


\section{Main Results}

In this section, we first intoduce congruence relations into formal contexts to obtain the relationships between congruence relations and the corresponding object oriented concept lattices. And then a unified model of formal contexts and formal decision contexts is constructed, which is called a consistent approximate representation space. It is proved that formal contexts and formal decision contexts are special cases of the consistent approximate representation space.

\subsection{Relationships between Congruence Relations and the Corresponding Object Oriented Concept Lattices}

In this subsection, we first give the definition of a congruence relation based on formal contexts, and then obtain the relationships between congruence relations and the corresponding object oriented concept lattices.

Let $(U, A, I)$ be a formal context. For $B \subseteq A$, we define a binary relation on the object power set $P(U)$ as follows:

$$
R^{\square B}=\left\{(X, Y) \in P(U) \times P(U) \mid X^{\square B}=Y^{\square B}\right\} .
$$

It is obvious that for any $B \subseteq A, R^{\square B}$ is a congruence relation on $(P(U), \cap)$ and $\left(U, R^{\square B}\right)$ is a dependence space according to Proposition 1 . We then define $[X]_{R^{\square} B}=\left\{Y \in P(U) \mid(X, Y) \in R^{\square B}\right\}$ the congruence class respect to $X$, and $I N_{R^{\square}}(X)=\cap\left\{Y \mid Y \in[X]_{R^{\square B}}\right\}$.

Lemma 1 Let $(U, A, I)$ be a formal context. For $X, Y, Z \subseteq U$ and $B \subseteq A$, the following statements hold:

(1) $\left(I N_{R^{\square}}^{\square_{B}}(X), X\right) \in R^{\square B}$,

(2) $I N{ }_{R^{\square} B}(X)$ is an inner operator,

(3) If $X \subseteq Y \subseteq Z$ and $(X, Z) \in R^{\square B}$, then $(X, Y) \in R^{\square B}$ and $(Y, Z) \in R^{\square B}$.

Proof. (1) Since $\left(I N_{R^{\square} B}(X)\right)^{\square_{B}}=\left(\cap\left\{Y \mid Y \in[X]_{R^{\square_{B}}}\right\}\right)^{\square_{B}}=\underset{Y \in[X]_{R^{\square} B}}{\cap} Y^{\square_{B}}=X^{\square_{B}}$ holds by Proposition 1, we have $\left(I N{ }_{R^{\square} B}(X), X\right) \in R^{\square_{B}^{B}}$.

(2) In order to prove that $I N_{{ }^{\square}}(X)$ is an inner operator, we should prove that (a) $I N_{R^{\square} B}(X) \subseteq X$ for any $X \subseteq U$; (b) if $X \subseteq Y$, then $I N_{R^{\square} B}(X) \subseteq I N_{R^{\square} B}(Y)$; and (c) $I N_{R^{\square}}(X)=I N_{R^{\square}}\left(I N_{R^{\square}}(X)\right)$

Obviously, (a) $I N_{R^{\square_{B}}}(X) \subseteq X$ holds by the definition of $I N_{R^{\square_{B}}}(X)$. (b) Since $\left(I N_{R^{\square} B}(X), X\right) \in R^{\square_{B}}$ and $\left(I N_{R^{\square} B}(Y), Y\right) \in R^{\square_{B}^{B}}$ hold for any $X$ and $Y$, we have $\left(I N_{R^{\square} B}(X) \cap I N_{R^{\square}}(Y), X \cap Y\right) \in R^{\square_{B}^{B}}$ according to Definition 4. Thus, if $X \subseteq Y$, then $\quad\left(I N_{R^{\square} B}(X) \cap I N_{R^{\square} B}(Y), X\right) \in R^{\square_{B}} \quad$ holds which shows that $I N_{R^{\square} B}(X) \cap I N_{R^{\square} B}(Y)=I N_{R^{\square B}}(X)$. Therefore, $I N_{R^{\square} B}(X) \subseteq I N_{R^{\square}}(Y)$. (c) Finally, since $\left(I N_{R}^{\square_{B}}(X), X\right) \in R^{\square_{B}} \quad$ and $\quad\left(I N_{R^{\square B}}(X), I N_{R^{\square} B}\left(I N_{R^{\square} B}(X)\right)\right) \in R^{\square_{B} B} \quad, \quad$ we have 
$\left(X, I N_{R^{\square} B}\left(I N_{R^{\square} B}(X)\right)\right) \in R^{\square_{B}} \quad$, which leads $\quad$ to $\quad I N_{R^{\square} B}(X) \subseteq I N_{R^{\square} B}\left(I N_{R^{\square} B}(X)\right)$. So $I N_{R^{\square} B}(X)=I N_{R^{\square} B}\left(I N_{R^{\square} B}(X)\right)$ holds.

(3) Since $(Y, Y) \in R^{\square_{B}}$ and $(X, Z) \in R^{\square_{B}}$, we have $(X \cap Y, Z \cap Y) \in R^{\square_{B}}$. So if $X \subseteq Y \subseteq Z$, then we have $(X, Y) \in R^{\square_{B}}$. Combining $(X, Z) \in R^{\square_{B}}$ and $(X, Y) \in R^{\square^{B}}$, we have $(Y, Z) \in R^{\square^{B}}$.

Lemma 1 shows that $I N_{R^{\square B}}(X)$ is the minmum element in $[X]_{R^{\square} B}$. An object subset $X \subseteq U$ is called $I N_{R^{\square B}}$-closed if $I N_{R^{\square B}}(X)=X$. The set of all $I N_{R^{\square} B}$-closed sets is denoted by $\Pi_{B}$.

Lemma 2 Let $(U, A, I)$ be a formal context. For $X \subseteq U$ and $B \subseteq A$, we have

(1) $I N_{R^{\square} B}(X)=X^{\square B \diamond B}$.

(2) $\Pi_{B}=L_{O U}\left(U, B, I_{B}\right)$.

(3) $\left(I N_{R^{\square} B}(X), X^{\square B}\right) \in L_{O U}\left(U, B, I_{B}\right)$.

Proof. (1) By Proposition 1 and Lemma 1, we have $X^{\square_{B \diamond B}}=\left(I N_{R^{\square_{B}}}(X)\right)^{\square_{B} \otimes_{B}} \subseteq I N_{R^{\square_{B}}}(X)$. Conversely, $X^{\square_{B \diamond B} \square_{B}}=X^{\square_{B}}$ by Proposition 1. So $X^{\square_{B} \diamond B} \in[X]_{R^{\square B}}$. Then, $I N_{R^{\square} B}(X) \subseteq X^{\square_{B} \diamond_{B}}$. Therefore, $I N_{R^{\square}}(X)=X^{\square B \diamond B}$.

(2) For any $X \in L_{O U}\left(U, B, I_{B}\right)$, it is clear that $X=X^{\square \otimes \diamond B}$. Then, by (1), we have $I N_{R^{\square_{B}}}(X)=X^{\square_{B} \diamond B}=X$, i.e., $X$ is a $I N_{R^{\square_{B}}}$-closed set. Therefore, $L_{O U}\left(U, B, I_{B}\right) \subseteq \Pi_{B}$. Furthermore, for any $X \in \Pi_{B}$, we have $I N_{R^{\square_{B}}}(X)=X$. Using (1) again, $I N_{R^{\square} B}(X)=X^{\square_{B} \diamond B}=X$, that is $X \in L_{O U}\left(U, B, I_{B}\right)$. Therefore we conclude $\Pi_{B} \subseteq L_{O U}\left(U, B, I_{B}\right)$.

(3) follows immediately from (1) and Lemma 1.

Lemma 2 says that all the $I N_{R^{\square}}$-closed sets form the extent set of $\left(U, B, I_{B}\right)$ exactly.

Lemma 3 Let $\left(U, A_{1}, I_{1}\right)$ and $\left(U, A_{2}, I_{2}\right)$ be two formal contexts with the same object set. If $L_{O U}\left(U, A_{2}, I_{2}\right) \subseteq L_{O U}\left(U, A_{1}, I_{1}\right)$, for $X \subseteq U$, we have the following two statements:

(1) $I N_{R}^{\square A_{1}}\left(I N{ }_{R}^{\square A_{2}}(X)\right)=I N{ }_{R}^{\square A_{2}}(X)$,

(2) $I N_{R^{\square} A_{2}}(X) \subseteq I N{ }_{R}^{\square A_{1}}(X)$.

Proof. (1) Since $L_{O U}\left(U, A_{2}, I_{2}\right) \subseteq L_{O U}\left(U, A_{1}, I_{1}\right)$ and for $X \subseteq U$ $I N_{R^{\square} A_{2}}(X) \in L_{O U}\left(U, A_{2}, I_{A_{2}}\right)$ by Lemma 2, we have $I N_{R^{\square} A_{2}}(X) \in L_{O U}\left(U, A_{1}, I_{A_{1}}\right)$ and $\left(I N_{R^{\square} A_{2}}(X)\right)^{\square A_{1} \triangleright A_{1}}=I N_{R^{\square} A_{2}}(X)$. Combined with $\left(I N_{R^{\square} A_{2}}(X)\right)^{\square A_{1} \triangleright A_{1}}=I N_{R^{\square} A_{1}}\left(I N_{R^{\square} A_{2}}(X)\right)$, (i) is concluded.

(2) Since $I N_{R} A_{A_{1}}$ and $I N_{R} A_{A_{2}}(X)$ are two inner operators, we have $I N_{R}^{\square A_{2}}(X) \subseteq X$ and $I N{ }_{R}^{\square A_{1}}\left(I N{ }_{R^{\square} A_{2}}(X)\right) \subseteq I N{ }_{R}^{\square A_{1}}(X)$. Thus, $I N_{R^{\square} A_{2}}(X) \subseteq I N_{\mathbb{D}^{\square} A_{1}}(X)$ follows directly from (i). 
Theorem 1 Let $\left(U, A_{1}, I_{1}\right)$ and $\left(U, A_{2}, I_{2}\right)$ be two formal contexts with the same object set. Then we have, $L_{\text {OU }}\left(U, A_{2}, I_{2}\right) \subseteq L_{O U}\left(U, A_{1}, I_{1}\right) \Leftrightarrow R^{\square A_{1}} \subseteq R^{\square A_{2}}$.

Proof. Sufficiency. Assume $L_{O U}\left(U, A_{2}, I_{2}\right) \subseteq L_{O U}\left(U, A_{1}, I_{1}\right)$ does not hold, then there exists $X \in L_{O U}\left(U, A_{2}, I_{2}\right)$ such that $X \notin L_{O U}\left(U, A_{1}, I_{1}\right)$. Thus, $I N{ }_{R} A_{A_{1}}(X) \subset X=I N_{R} \square_{A_{2}}(X)$ is concluded by Lemma 1. Since $R^{\square A_{1}} \subseteq R^{\square A_{2}}$ implies $[X]_{R^{D_{1}}} \subseteq[X]_{R^{\square A_{2}}}$, we have $I N{ }_{R}^{\square A_{2}}(X) \subseteq I N_{R} A_{A_{1}}(X)$, which is a contradiction to $I N_{R}^{\square A_{1}}(X) \subset I N_{R} A_{A_{2}}(X)$. Consequently, $L_{O U}\left(U, A_{2}, I_{2}\right) \subseteq L_{O U}\left(U, A_{1}, I_{1}\right)$.

Necessity. Assume $R^{\square A_{1}} \subseteq R^{\square A_{2}}$ does not hold, then there exits $X \subseteq U$ such that $[X]_{R^{\square \Lambda_{1}}} \subseteq[X]_{R^{\square \Lambda_{2}}}$ does not hold. Thus, there exists $Y \in[X]_{R^{0 \Lambda_{1}}}$ such that $Y \notin[X]_{R^{0 \Lambda_{2}}}$. We prove it from two cases: $X \in L_{O U}\left(U, A_{1}, I_{1}\right)$ and $X \notin L_{O U}\left(U, A_{1}, I_{1}\right)$.

Firstly, we suppose $X \in L_{O U}\left(U, A_{1}, I_{1}\right)$. Since $Y \in[X]_{R^{0} A_{1}}$ and $Y \notin[X]_{R^{0} \Lambda_{2}}$, we obtain $X=I N{ }_{R}^{\square A_{1}}(Y) \subset Y$. Combining with $I N_{R^{D} A_{2}}(Y) \subseteq I N_{R^{\square} A_{1}}(Y)$ by Lemma 3, we have $I N_{R^{\square} A_{2}}(Y) \subseteq X \subset Y$. Due to Lemma $1,(Y, X) \in R^{\square A_{2}}$, which is a contradiction to $Y \notin[X]_{R^{\square} A_{2}}$. Therefore, $R^{\square A_{1}} \subseteq R^{\square A_{2}}$ holds.

Secondly, we suppose $X \notin L_{O U}\left(U, A_{1}, I_{1}\right)$. According to the above discussions, we have $\left[I N{ }_{R}^{\square_{A_{1}}}(X)\right]_{R}{A_{A}}_{A_{1}} \subseteq\left[I N{ }_{R}^{\square_{A_{1}}}(X)\right]_{R} \square_{A_{2}}$ due to $I N_{R} \square_{A_{1}}(X) \in L_{O U}\left(U, A_{1}, I_{1}\right)$. Since $Y \in[X]_{R^{D_{A}}}$, it is evident that $I N_{R}{ }_{D_{1}}(X) \subseteq Y \quad$ and $\quad Y \in\left[I N_{R}{ }_{A_{1}}(X)\right]_{R}{ }_{R} A_{2}$. Combining with $I N{ }_{R}^{\square A_{2}}(X) \subseteq I N{ }_{R}^{\square} A_{1}(X)$ we have $I N_{R}^{\square A_{2}}(X) \subseteq Y$. Since $Y \in\left[I N_{R} \mathbb{D}_{A_{1}}(X)\right]_{R} \square_{A_{2}}$, we obtain $I N \underset{R}{\square A_{2}}\left(I N{ }_{R}^{\square A_{1}}(X)\right)=I N_{R}^{\square A_{2}}(Y) \quad$. According $\quad$ to $\quad I N_{R}^{\square A_{1}}(X) \subseteq X \quad$, we have $I N \underset{R}{\square A_{2}}\left(I N{ }_{R}^{\square A_{1}}(X)\right) \subseteq I N \underset{R}{\square A_{2}}(X)$. Thus, $I N_{R}^{\square A_{2}}(Y) \subseteq I N_{R}^{\square A_{2}}(X) \subseteq Y$. By Lemma 1, ( IN $\left.{ }_{R}^{\square A_{2}}(X), Y\right) \in R^{\square A_{2}}$ holds. That is, $(X, Y) \in R^{\square A_{2}}$, which is a contradiction to $Y \notin[X]_{R^{\square} A_{2}}$.

Therefore, $R^{\square A_{1}} \subseteq R^{\square A_{2}}$ is concluded.

Consequently, if $L_{O U}\left(U, A_{2}, I_{2}\right) \subseteq L_{O U}\left(U, A_{1}, I_{1}\right)$, then $R{ }^{\square A_{1}} \subseteq R^{\square A_{2}}$ holds.

\subsection{Consistent Approximate Representation Space for Formal Contexts and Formal Decision Contexts}

In this subsection, we define the consistent approximate representation space to unify formal contexts and formal decision contexts.

Definition 5 Let $(U, A, I)$ be a formal context. $\Re^{\square}=\left\{R^{\square\{a\}} \subseteq P(U) \times P(U) \mid a \in A\right\}$ is a family of equivalence relations on $P(U)$ and $R^{\prime}$ is an equivalence relation on $P(U)$. A quadruple $\left(U, A, \Re^{\square}, R^{\prime}\right)$ is said to be an approximate representation space of the context $(U, A, I)$. 
Definition 6 Let $S=\left(U, A, \Re^{\square}, R^{\prime}\right)$ be a consistent approximate representation space and $R^{\square B}=\underset{a \in B}{\cap} R^{\square\{a\rangle}$ for any $B \subseteq A$. If $R^{\square A} \subseteq R^{\prime}$, then $S$ is called a consistent approximate representation space of the context $(U, A, I)$.

Obviously, $S=\left(U, A, \mathfrak{R}^{\square}, R^{\square}\right)$ is a consistent approximate representation space of $(U, A, I)$. In fact, for any $a \in A, R^{\square\{a\rangle}$ and $R^{\square A}$ are both equivalence relations on $P(U)$ and $R^{\square A} \subseteq R^{\square_{A}}$, therefore $S=\left(U, A, \Re^{\square}, R^{A}\right)$ is a consistent approximate representation space of $(U, A, I)$ according to Definition 6.

Definition 7 Let $(U, A, I)$ and $(U, C, J)$ be two formal contexts with the same object set. $(U, A, I, C, J)$ is called a formal decision context, where $I \subseteq U \times A, J \subseteq U \times C$ and $A \cap C=\varnothing . A$ and $C$ are called condition attribute set and decision attribute set respectively.

Definition 8 Let $(U, A, I, C, J)$ be a formal decision context. $(U, A, I, C, J)$ is said to be consistent if $R^{\square A} \subseteq R^{\square c}$, otherwise, it is said to be inconsistent. Where $R^{\square C}=\left\{(X, Y) \in P(U) \times P(U) \mid X^{\square C}=Y^{\square C}\right\}$.

Combining Theorem 1 and Definition 8, we have $(U, A, I, C, J)$ is consistent if and only if $L_{O U}(U, C, J) \subseteq L_{O U}(U, A, I)$. So we have the following result directly.

Theorem 2 Let $(U, A, I, C, J)$ be a formal decision context and $B \subseteq A$. Then $S=\left(U, A, \Re^{\square}, R^{\square C}\right)$ is a consistent approximate representation space of $(U, A, I, C, J)$ iff the formal decision context $(U, A, I, C, J)$ is consistent.

In the following text, we will develop the notions of consistent approximate representation space based on inconsistent formal decision context.

Let $(U, A, I, C, J)$ be an inconsistent formal decision context. $R^{\square c}$ partitions $U$ into a family of disjoint subsets $U / R^{\square C}$, we denote $U / R^{\square C}=\left\{D_{1}, D_{2}, \ldots, D_{t}\right\}$, where $D_{j}, 1 \leq j \leq t$ is the decision congruence class. For any $X \in P(U), B \subseteq A$ and $D_{j} \in U / R^{\square} C, 1 \leq j \leq t$, we define $P\left(D_{j} /[X]_{R^{\square B}}\right)=\frac{\left|D_{j} \cap[X]_{R^{\square B}}\right|}{\left|[X]_{R^{\square B}}\right|}$, the degree in which the condition congruence class $[X]_{R^{0_{B}}}$ belongs to the decision congruence class $D_{j}$.

A membership distribution function $\mu_{B}: P(U) \rightarrow[0,1]$ is defined as follows:

$$
\mu_{B}(X)=\left(P\left(D_{1} /[X]_{R^{\square B}}\right), P\left(D_{2} /[X]_{R^{\square B}}\right), \ldots, P\left(D_{t} /[X]_{R^{\square B}}\right)\right) \text {. }
$$

Evidently, $\mu_{B}(X)$ is a conditional probability distribution on $U / R^{\square C}$. For any $X \in P(U)$, we denote the maximum decision function by

$$
\eta_{B}(X)=\left\{D_{j 0} \mid P\left(D_{j 0} /[X]_{R^{D B}}\right)=\max _{1 \leq j \leq t} P\left(D_{j} /[X]_{R^{\square B}}\right)\right\} .
$$

Theorem 3 Let $(U, A, I, C, J)$ be an inconsistent formal decision context and $B \subseteq A$. And we denote $R^{\square}=\left\{(X, Y) \in P(U) \times P(U) \mid \mu_{A}(X)=\mu_{A}(Y)\right\}$ and 


$$
R^{\square}=\left\{(X, Y) \in P(U) \times P(U) \mid \eta_{A}(X)=\eta_{A}(Y)\right\} .
$$

Then $S_{\mu}=\left(U, A, \mathfrak{R}^{\square}, R^{\square}\right)$ and $S_{\eta}=\left(U, A, \mathfrak{R}^{\square}, R^{\square}\right)$ are both consistent approximate representation spaces of $(U, A, I, C, J)$. And we call $S_{\mu}=\left(U, A, \mathfrak{R}^{\square}, R^{\square}\right)$ the distribution consistent approximate representation spaces, and $S_{\eta}=\left(U, A, \mathfrak{R}^{\square}, R^{\square}\right)$ the maximum decision consistent approximate representation spaces of $(U, A, I, C, J)$.

For any $B \subseteq A$, the lower and upper approximation distribution functions of inconsistent formal decision context $(U, A, I, C, J)$ are defined as follows:

$$
\begin{aligned}
& \underline{R}^{\square B}=\left(\underline{R}^{\square B}\left(D_{1}\right), \underline{R}^{\square B}\left(D_{2}\right), \ldots, \underline{R}^{\square B}\left(D_{t}\right)\right), \\
& \bar{R}^{\square B}=\left(\bar{R}^{\square B}\left(D_{1}\right), \bar{R}^{\square}\left(D_{2}\right), \ldots, \bar{R}^{\square_{B}}\left(D_{t}\right)\right),
\end{aligned}
$$

Where $\underline{R}^{\square B}\left(D_{j}\right)=\left\{[X]_{R^{\square B}} \mid[X]_{R^{\square B}} \subseteq D_{j}\right\}, \bar{R}^{\square B}\left(D_{j}\right)=\left\{[X]_{R^{\square B}} \mid[X]_{R^{\square B}} \cap D_{j} \neq \varnothing\right\}$.

The set of the condition congruence classes which belong to the decision congruence class $D_{j}$ is determined by $\underline{R}^{\square B}\left(D_{j}\right)$, while $\bar{R}^{\square}\left(D_{j}\right)$ is the set of the condition congruence classes which possibly belong to $D_{j}$.

For any $B \subseteq A$ and $X \in P(U)$, we denote

$$
\begin{gathered}
G_{B}(X)=\left\{D_{j} \in U / R^{\square C} \mid[X]_{R^{\square B}} \subseteq D_{j}\right\}, \\
M_{B}(X)=\left\{D_{j} \in U / R^{\square C} \mid[X]_{R^{\square B}} \cap D_{j} \neq \varnothing\right\} .
\end{gathered}
$$

Theorem 4 Let $(U, A, I, C, J)$ be an inconsistent formal decision context and $B \subseteq A$. And we denote

$$
\begin{aligned}
& \underline{R}^{\square}=\left\{(X, Y) \in P(U) \times P(U) \mid G_{A}(X)=G_{A}(Y)\right\} \\
& \bar{R}=\left\{(X, Y) \in P(U) \times P(U) \mid M_{A}(X)=M_{A}(Y)\right\}
\end{aligned}
$$

Then $\underline{S}=\left(U, A, \mathfrak{R}^{\square}, \underline{R}^{\square}\right) \quad$ and $\bar{S}=\left(U, A, \mathfrak{R}^{\square}, \bar{R}\right)$ are both consistent approximate representation spaces of $(U, A, I, C, J)$. And we call $\underline{S}=(U, A, \mathfrak{R}, \underline{R})$ the lower consistent approximate representation spaces, and $\bar{S}=(U, A, \mathfrak{R}, \bar{R})$ the upper consistent approximate representation spaces of $(U, A, I, C, J)$.

Suppose $S=\left(U, A, \Re^{\square}, R^{\prime}\right)$ is a consistent approximate representation space. According to Theorem 2-4, we obtain the following results:

(i) If $R^{\prime}=R^{\square}$, then $S=\left(U, A, \Re^{\square}, R^{\prime}\right)$ can be regarded as the consistent approximate representation space of the context $(U, A, I)$.

(ii) If $R^{\prime}=R^{\square C}$, then $S=\left(U, A, \Re^{\square}, R^{\prime}\right)$ can be regarded as the consistent approximate representation space of the consistent formal decision context $(U, A, I, C, J)$.

(iii) If $R^{\prime}=R^{\square}$, then $S=\left(U, A, \Re^{\square}, R^{\prime}\right)$ can be regarded as the distribution consistent approximate representation space of the inconsistent formal decision context $(U, A, I, C, J)$. 
(iv) If $R^{\prime}=R^{\square}$, then $S=\left(U, A, \Re^{\square}, R^{\prime}\right)$ can be regarded as the maximum decision consistent approximate representation space of the inconsistent formal decision context $(U, A, I, C, J)$.

(v) If $R^{\prime}=\underline{R}^{\square}$, then $S=\left(U, A, \Re^{\square}, R^{\prime}\right)$ can be regarded as the lower consistent approximate representation space of the inconsistent formal decision context $(U, A, I, C, J)$.

(vi) If $R^{\prime}=\bar{R}$, then $S=\left(U, A, \Re^{\square}, R^{\prime}\right)$ can be regarded as the supper consistent approximate representation space of the inconsistent formal decision context $(U, A, I, C, J)$.

Therefore, formal contexts and formal decision contexts have the unified form consistent approximate representation space. Furthermore, we can obtain knowledge on reduction and rule acquisition of formal contexts and formal decision contexts through discussing the corresponding results in consistent approximate representation space.

\section{Conclusion}

This paper has developed the notion of consistent approximate representation space in order to construct the unified model of formal contexts and formal decision contexts based on object oriented concept lattices. It is shown that formal contexts and formal decision contexts can be regarded as special cases of consistent approximate representation space. In further research, we will study knowledge reduction and rule acquisition of consistent approximate representation space based on object oriented concept lattices in order to obtain the corresponding results in formal contexts and formal decision contexts, which can reduce the complexities of different contexts.

\section{Acknowledgements}

The authors gratefully acknowledge the support of the Natural Science Foundation of China (Nos. 61202206, 61272021, 61075120, 61173181, 11071284, 60673096 and 11071281) and the Natural Science Foundation of Zhejiang Province (No. LZ12F03002).

\section{References}

[1] R. Wille, "Restructuring lattice theory: an approach based on hierarchies of concepts", I. Rival (Ed.), Ordered Sets, Reidel, Dordrecht-Boston, (1982), pp. 445-470.

[2] B. Ganter and R. Wille, "Formal Concept Analysis: Mathematical Foundations", Springer-Verlag, New York, (1999).

[3] T. B. Ho, "An approach to concept formation based on formal concept analysis", IEICE Trans. Information and Systems. E78-D, vol. 5, (1995), pp. 553-559.

[4] C. Carpineto and G. Romano, "Galois: An order-theoretic approach to conceptual clustering", Proceedings of ICML, vol. 293, (1993), pp. 33-40.

[5] R. Godin, "Incremental concept formation algorithm based on Galois (concept) lattices", Computational Intelligence, vol. 11, no. 2, (1995), pp. 246-267.

[6] G. D. Oosthuizen, "The application of concept lattice to machine learning", Technical Report, University of Pretoria, South Africa, (1996).

[7] J. Saquer and J. Deogun, "Formal rough concept analysis", Proceedings of the 7th International Workshop on New Directions in Rough Sets, Data Mining, and Granular-Soft Computing, LNCS 1711, Springer, Berlin, (1999), pp. 91-99.

[8] J. Saquer and J. Deogun, "Concept approximations based on rough sets and similarity measures", International Journal of Applied Mathematics and Computer Science, vol. 11, (2001), pp. 655-674.

[9] K. Hu, Y. Sui, Y. Lu, J. Wang and C. Shi, "Concept approximation in concept lattice", Proceedings of 5th Pacific-Asia Conference, PAKDD'01, Lecture Notes in Computer Science, (2001), pp. 167-173. 
[10] Y. Y. Yao, "Rough set approximations in formal concept analysis", Proceedings of 2004 Annual Meeting of the North American Fuzzy Information Processing Society, (2004), pp. 73-78.

[11] G. Gediga and I. Duntsch, "Modal-style operators in qualitative data analysis", Proceedings of the 2002 IEEE International Conference on Data Mining, (2002), pp. 155-162.

[12] Y. Y. Yao, "A comparative study of formal concept analysis and rough set theory in data analysis", Proceedings of 3rd International Conference, RSCTC'04, (2004), pp. 59-68.

[13] Y. Y. Yao, "Concept lattices in rough set theory", Proceedings of $23^{\text {rd }}$ International Meeting of the North American Fuzzy Information Processing Society, (2004), pp. 796-801.

[14] P. A. Grigoriev and S. A. Yevtushenko, "Elements of an agile discovery environment", Proceedings of 6th International Conference on Discovery Science, Lecture Notes in Artificial Intelligence, vol. 2843, (2003), pp. 309-316.

[15] M. Liu, M. W. Shao, W. X. Zhang and C. Wu, "Reduction method for concept lattices based on rough set theory and its application", Computers and Mathematics with Applications, vol. 53, (2007), pp. 1390-1410.

[16] X. Wang and W. X. Zhang, "Relations of attribute reduction between object and property oriented concept lattices", Knowledge-Based Systems, vol. 21, no. 5, (2008), pp. 398-403.

[17] W. X. Zhang, L. Wei and J. J. Qi, "Attribute reduction theory and approach to concept lattice", Science in China Series F-Information Science, vol. 48, no. 6, (2005), pp. 713-726.

[18] W. X. Zhang, L. Wei and J. J. Qi, "Attribute reduction in concept lattice based on discernibility matrix", Proceedings of RSFDGrC 2005, Lecture Notes in Artificial Intelligence, (2005), pp. 157-165.

[19] W. X. Zhang and G. F Qiu, "Uncertain Decision Making Based on Rough Sets", Tsinghua University Publishing House, (2005).

[20] L. Wei, J. J. Qi and W. X. Zhang, "Attribute reduction theory of concept lattice based on decision formal contexts", Science in China Series F-Information Science, vol. 51, no. 7, (2008), pp. 910-923.

[21] X. Wang and J. M. Ma, "A novel approach to attribute reduction in concept lattices", Proceedings of RSKT 2006, Lecture Notes in Artificial Intelligence, (2006), pp. 522-529.

[22] W. Z. Wu, Y. Leung and J. S. Mi, "Granular computing and knowledge reduction in formal contexts", IEEE Transactions Knowledge and Data Engineering, vol. 10, no. 21, (2009), pp. 1461-1474.

[23] H. W. Liu, L. Liu and H. J. Zhang, "A fast pruning redundant rule method using Galois connection”, Applied Soft Computing, vol. 11, (2011), pp. 130-137.

[24] J. S. Mi, Y. Leung and W. Z. Wu, "Approaches to attribute reduction in concept lattices induced by axialities", Knowledge-Based Systems, vol. 23, (2010), pp. 504-511.

[25] J. H. Li, C. L. Mei and Y. J. Lv, "Knowledge reduction in real decision formal contexts", Information Sciences, vol. 189, (2012), pp. 191-207.

[26] C. A. Kumar and S. Srinivas, "Concept lattice reduction using fuzzy K-means clustering", Expert Systems with Applications, vol. 37, (2010), pp. 2696-2704.

[27] X. Wang, "Approaches to attribute reduction in concept lattices based on rough set theory", International Journal of Hybrid Information Technology, vol. 5, no. 2, (2012), pp. 67-80.

[28] X. Wang and W. Z. Wu, "Approximate reduction in inconsistent formal decision contexts", 2012 IEEE International Conference on Granular Computing, (2012), pp. 616-621.

[29] M. Novotny, "Dependence spaces of information system", E. Orlowska (Ed.), Incomplete Information: Rough Set Analysis, Physica-Verlag, Heidelberg-New York, (1998), pp. 193-246. 